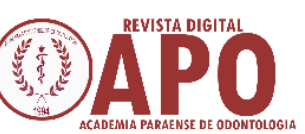

\title{
Morfologia foraminal após utilização de sistemas rotatórios para patência apical
}

\author{
Morphology of the apical foramen after use of the rotatory system for \\ apical patency
}

\author{
Bruna Araújo Smith ${ }^{1}$ \\ Débora Ferreira Monteiro da Silva ${ }^{2}$ \\ Juliana do Socorro Moraes Melo ${ }^{2}$ \\ Roberta Fonseca de Castro ${ }^{3}$ \\ Luis Carlos de Lima Dias Junior ${ }^{4}$ \\ Juliana Melo da Silva Brandão ${ }^{5}$
}

\section{RESUMO}

O objetivo deste estudo foi avaliar as alterações morfológicas do forame apical após patência apical de canais com diferentes sistemas rotatórios. Foram selecionadas trinta raízes de dentes humanos extraídos. As amostras foram divididas de forma aleatória em dois grupos $(n=15)$ de acordo com o sistema de instrumentação a ser utilizado. As do grupo 1 foram preparadas com instrumento rotatório PathFile 0.19 (Dentsply) e as do grupo 2 com instrumento Easy 25.01 (Easy). Todos os grupos foram preparados com o nível de instrumentação no limite do forame apical. Fotomicrografias foram realizadas em microscopia eletrônica de varredura (MEV), para registro da anatomia foraminal antes da instrumentação, e após a utilização de cada instrumento. Em seguida as 2 micrografias foram sobrepostas e o forame dividido em 4 quadrantes a partir do seu centro para comparação e análise. O grupo 2 apresentou maior desvio quando comparado com o grupo 1 ( $\mathrm{p}<0,05)$. No entanto, não houve diferença estatisticamente significante $(\mathrm{p}>0,05)$ entre os grupos 1 e 2 quanto ao alargamento foraminal. Nas condições do presente estudo, foi possível observar um menor desvio apical para o grupo no qual foi realizada a instrumentação com a lima PathFile e não houve diferença quanto ao alargamento foraminal entre os grupos analisados.

Palavras-chave: Endodontia. Canal radicular. Ápice dentário.

\begin{abstract}
The aim of this study was to evaluate the morphological changes of the apical foramen after apical patency with different rotary systems. Thirty roots of extracted human teeth were selected. The samples were divided randomly into two groups $(n=15)$ according to the instrumentation system to be used. Group 1 was prepared with rotary instruments Pathfile 0:19 (Dentsply) and group 2 with Easy 25.01 (Easy). All groups were prepared with the instrumentation level in the apical foramen limit. Photomicrographs were held in scanning electron microscopy (SEM), to record the foraminal anatomy before instrumentation, and after the use of each instrument. Then the two micrographs were superposed and divided into 4 quadrants foramen from its center. Group 2 had higher deviation when compared with group $1(\mathrm{p}<0.05)$. However, there was no statistically significant difference $(\mathrm{p}>0.05)$ between groups 1 and 2 about foraminal enlargement. Under the conditions of this study, we observed a lower apical to the group in which it was carried out with the instrumentation Pathfile and there was no difference in the foraminal enlargement among the groups.
\end{abstract}

Keyword: Endodontics. Root canal. Tooth apex.

\footnotetext{
${ }^{1}$ Graduanda, Faculdade de Odontologia, Universidade Federal do Pará

${ }^{2}$ Cirurgiã Dentista

${ }^{3}$ Mestre em Odontologia, Universidade Federal do Pará

${ }^{4}$ Mestrando, Odontologia, Universidade Federal do Pará

${ }^{5}$ Professora Doutora, Faculdade de Odontologia, Universidade Federal do Pará
} 


\section{INTRODUÇÃo}

Um dos principais objetivos do tratamento endodôntico é minimizar a quantidade de microrganismos do sistema de canais radiculares. $\mathrm{O}$ terço apical do canal radicular é considerado uma zona crítica, pois pode abrigar um grande número de microorganismos que irão perpetuar a inflamação perirradicular ${ }^{1,2}$.

A constrição apical (junção cemento-dentina) tem sido defendida como a parte final de instrumentação e obturação, sendo o final do canal dentinário e início do canal cementário. É a porção mais estreita do canal radicular, e ponto de encontro entre polpa $\mathrm{e}$ periodonto ${ }^{1,3}$.

O limite apical de instrumentação consiste em um tema controverso no campo da endodontia. Segundo a escola conservadora, o limite de trabalho do endodontista seria a constrição apical, $1 \mathrm{a} 2 \mathrm{~mm}$ aquém do forame apical. No entanto, a região apical é considerada um nicho para a colonização de bactérias. E para obter uma melhor remoção microbiana, é necessário que os canais sejam ampliados na região apical, proporcionando uma maior penetração das substâncias irrigadoras nesta região, que irá favorecer um melhor prognóstico para o tratamento endodôntico ${ }^{4,5}$.

A presença de micro-organismos e toxinas nos canais radiculares é a principal causa de danos à polpa, tecidos perirradiculares, persistência de patologias pulpares e periapicais. Portanto, esse fato pode ser evitado com a execução da patência foraminal durante os procedimentos de limpeza e modelagem ${ }^{6,7}$.

A patência é uma técnica na qual a porção apical do canal é mantida livre de detritos através da recapitulação com um instrumento na região foraminal. Os instrumentos utilizados para obter a patência são os mesmos utilizados inicialmente para exploração dos canais radiculares $8,9,10$.

O objetivo mecânico da patência apical visa a manutenção do terço apical livre de debris. Além do mais, do ponto de vista biológico, a patência visa que o forame permaneça livre de micro-organismos, diminuindo a infecção constituída no canal cementário $^{11,12}$.
Durante muito tempo, foram utilizadas somente as limas manuais de aço inoxidável para limpeza e modelagem dos canais radiculares, porém devido a menor flexibilidade das mesmas, foram introduzidas na endodontia os sistemas rotatórios de liga níquel-titânio (NiTi). Esses instrumentos rotatórios de NiTi, além de diminuírem as complicações clínicas, durante o preparo, como o bloqueio do canal, a formação de desvio, e perfuração; podem reduzir a fadiga do operador e o tempo necessário para a preparação do canal radicular ${ }^{13,14}$.

Entre as limas de NiTi utilizadas para a patência, estão as limas Glide Path $\left(\right.$ Easy $\left.{ }^{\circledR}\right)$ que é um sistema rotatório de NiTi composto por quatro instrumentos $(25.01,30.01,35.01,40.01)$ com a ponta do instrumento não-cônica e com uma secção transversal quadrada modificada $^{15,16}$.

Outro sistema de instrumentos rotatórios utilizado com a finalidade de realizar a patência apical e melhorar a configuração do preparo do canal radicular é o sistema PathFile (Dentsply®) composto de três instrumentos $(0.13,0.16$ e 0.19$)$ de secção transversal quadrangular e conicidade constante de $0,02^{17}$.

Diante do exposto, observou-se a necessidade de estudos que avaliem as possíveis alterações anatômicas no forame apical após a realização da patência. O trabalho teve como objetivo investigar a influência da patência apical, realizada com instrumentos rotatórios, na alteração da anatomia original (ampliação e desvio) do forame apical. A hipótese nula do estudo é de que não há diferença nas alterações anatômicas do forame entre os dois sistemas utilizados.

\section{Metodologia}

Para este experimento, foram utilizados 30 molares humanos extraídos, previamente à execução da pesquisa, armazenados em frasco único, sem identificação dos indivíduos dos quais os dentes foram extraídos. O projeto foi aprovado pelo Comitê de Ética em Pesquisa do ICS da Universidade Federal do Pará, parecer $\mathrm{n}^{\circ} 771.852$. Foram incluídos neste estudo molares superiores, sem cárie radicular visível, sem 
sinais de reabsorção ou calcificação, sem fraturas ou trincas e com formação apical completa.

Os dentes foram seccionados ao nível da junção amelocementária, utilizando-se discos flexíveis diamantados (KG Sorensen, São Paulo, SP, BR) em baixa rotação. Dessa forma, as raízes foram padronizadas em $12 \mathrm{~mm}$, com o auxílio de um paquímetro digital PD-150 (Vonder, Curitiba, PR, Brasil), sendo armazenadas em soro fisiológico. As raízes distovestibulares de molares superiores foram utilizadas para análise da anatomia foraminal.

As fotomicrografias foram realizadas antes que qualquer lima fosse utilizada no canal radicular e após a utilização das limas rotatórias, consequentemente obtiveram-se duas imagens de cada amostra.

As amostras foram divididas de forma aleatória em dois grupos $(\mathrm{n}=15)$ de acordo com o instrumento rotatório que realizou a patência. $\mathrm{O}$ comprimento de trabalho dos canais radiculares foi determinado por inspeção visual, introduzindo-se uma lima tipo $\mathrm{K}$ de calibre \#10 (Dentsply Maillefer, Ballaigues, CH), e registrando o comprimento desta no limite do ápice radicular.

Grupo 1 - PathFile: As raízes foram preparadas com instrumentos rotatórios PathFile 19.02 (Dentsply Maillefer $\left.{ }^{\circledR}\right)$. Antes do uso da lima rotatória, foi realizada exploração do canal com a lima manual tipo $\mathrm{K}$ \#10, e o comprimento de trabalho durante a instrumentação rotatória foi estabelecido no limite do forame apical, utilizando as limas rotatórias de patência selecionadas, de acordo com cada grupo.

Grupo 2 - Easy \#25.01: As raízes foram preparadas com instrumentos rotatórios Easy \# 25.01 (Easy, Belo Horizonte, MG, BR). A instrumentação e a modelagem dos canais foram realizadas de forma semelhante as do Grupo Path File (Dentsply®).

A instrumentação foi realizada por um único operador, com motor elétrico Easy (Easy®). Para o grupo Path File, foi estabelecida velocidade de $350 \mathrm{rpm}$ e torque de $1 \mathrm{~N}$ e para o Grupo Easy \#25.01, foi adotado velocidade de $350 \mathrm{rpm}$ e torque de $0.5 \mathrm{~N}$. As limas foram utilizadas de acordo com o proposto pelo fabricante para cada grupo.
Como substância auxiliar da instrumentação, foi utilizado 0,5mL de gel de Clorexidina a 2\% (Endogel, Essencial Pharma, Itapetininga, SP, BR) intercalada ao uso de $5 \mathrm{~mL}$ de soro fisiológico. Os canais foram irrigados com uma seringa plástica de $5 \mathrm{~mL}$ (Ultradent Ind. e Com. Ltda., Petrópolis, RJ, BR) e agulha de 30G (NaviTip, Ultradent Products Inc. South Jordan, UT).

Antes de serem levadas ao microscópio eletrônico de varredura (MEV), todas as amostras foram secadas com jato de ar e armazenadas em estufa a $37^{\circ} \mathrm{C}$ durante uma hora. A secagem foi suficiente para a análise dos forames apicais no $\mathrm{MEV}$ com $3 \mathrm{kV}$, dispensando a metalização dos espécimes. A plataforma móvel do MEV foi regulada para estar sempre na mesma posição inicial ajustando-se os eixos, $\mathrm{x}$, y e $\mathrm{z}$.

Para a análise ao MEV, os parâmetros específicos de $3 \mathrm{kV}$ e ampliação de 50x foram utilizados (JSM5600LV, JEOL, Tokyo, JP). Os espécimes foram montados em suportes de alumínio (stubs), com $12,7 \mathrm{~mm} \emptyset \times 3,1 \mathrm{~mm} \emptyset$, com os ápices voltados para cima, de maneira a serem avaliados e fotografados na mesma posição inicial. As amostras foram fixadas com fitas adesivas dupla face (3M, Sumaré, SP, BR), numeradas e identificadas, a fim de levá-las sempre na mesma posição no MEV.

As fotomicrografias foram realizadas antes que qualquer lima fosse utilizada no canal radicular e após a utilização das limas rotatórias, consequentemente obtiveram-se duas imagens de cada amostra.

A plataforma móvel do MEV foi regulada para estar sempre na mesma posição inicial. As 2 micrografias foram sobrepostas e o forame dividido em 4 quadrantes a partir do seu centro. Cada segmento foi avaliado de acordo com a metodologia proposta por Silva et al. ${ }^{18}$. A análise foi efetuada por dois examinadores treinados e calibrados para o estudo, a partir de um sistema de scores que variava de 0 a 4 , como observado na tabela 1 . Para o estudo, foi analisado um total de 30 imagens. 
Tabela 1: Scores utilizados para análise do alargamento foraminal e desvio da anatomia original.

\begin{tabular}{ccc}
\hline Score & $\begin{array}{c}\text { Alargamento do } \\
\text { forame apical }\end{array}$ & $\begin{array}{c}\text { Análise Morfológica } \\
\text { do Forame Apical }\end{array}$ \\
\hline 0 & Sem alargamento & Sem desvio \\
\hline 1 & $\begin{array}{c}\text { Alargamento de um } \\
\text { quarto }\end{array}$ & $\begin{array}{c}\text { Desvio de um } \\
\text { quarto }\end{array}$ \\
\hline 2 & $\begin{array}{c}\text { Alargamento de dois } \\
\text { quartos }\end{array}$ & $\begin{array}{c}\text { Desvio de dois } \\
\text { quartos }\end{array}$ \\
\hline 3 & $\begin{array}{c}\text { Alargamento de três } \\
\text { quartos }\end{array}$ & $\begin{array}{c}\text { Desvio de três } \\
\text { quartos }\end{array}$ \\
\hline 4 & Alargamento de quatro \\
quartos & $\begin{array}{c}\text { Desvio de quatro } \\
\text { quartos }\end{array}$ \\
\hline
\end{tabular}

Fonte: Silva et al, 2016.

\section{Resultados}

Para análise da concordância interexaminador, utilizou-se o teste Kappa ponderado. O resultado obtido para análise de alargamento e desvio foi de 0,66. O resultado encontra-se no intervalo de 0,61 a 0,80 , o que sugere que há uma concordância substancial.

Os grupos foram submetidos ao teste de Wilcoxon e Mann-Whitney, aplicados a um nível alfa de 5\%. Ao se comparar os valores da ampliação foraminal e desvio apical antes e após a utilização das limas de patência no grupo 1 e 2, observou-se diferença estatística $(\mathrm{p}<0,05)$ em ambos os grupos para o alargamento e desvio (Tabela 2). Ao comparar os grupos em relação ao desvio, o resultado foi significativamente maior $(\mathrm{p}>0,05)$ para o grupo 2. No entanto quanto ao alargamento foraminal, não houve diferença significativa $(p>0,05)$ entre os grupos analisados (Tabela 3). Na figura 1 observam-se imagens representativas dos diferentes grupos experimentais.

Tabela 2: Teste Wilcoxon para análise de desvio e alargamento após uso das limas de patência

\begin{tabular}{|c|c|c|c|c|c|c|c|c|}
\hline \multirow{2}{*}{ Grupo } & \multicolumn{4}{|c|}{ Alargamento } & \multicolumn{4}{|c|}{ Desvio } \\
\hline & $\mathrm{N}$ & Mediana & $Z$ & $\mathrm{p}$ & $\mathrm{N}$ & Mediana & Z & $\mathrm{p}$ \\
\hline 1 & 15 & 2 & & & 15 & 2 & & \\
\hline 2 & 15 & 2 & 1.55 & 0.1198 & 15 & 0 & 2.86 & 0.0042 \\
\hline
\end{tabular}

Tabela 3: Teste Wilcoxon para análise de desvio e alargamento após uso das limas de patência

\begin{tabular}{|c|c|c|c|c|c|c|c|c|}
\hline \multirow{2}{*}{ Grupo } & \multicolumn{4}{|c|}{ Alargamento } & \multicolumn{4}{|c|}{ Desvio } \\
\hline & $\mathrm{N}$ & Mediana & $Z$ & $\mathrm{p}$ & $\mathrm{N}$ & Mediana & $Z$ & $p$ \\
\hline 1 & 15 & 3 & 3.17 & 0.0015 & 15 & 3 & 3.05 & 0.0022 \\
\hline 2 & 15 & 3 & 2.80 & 0.0051 & 15 & 3 & 2.02 & 0.0431 \\
\hline
\end{tabular}

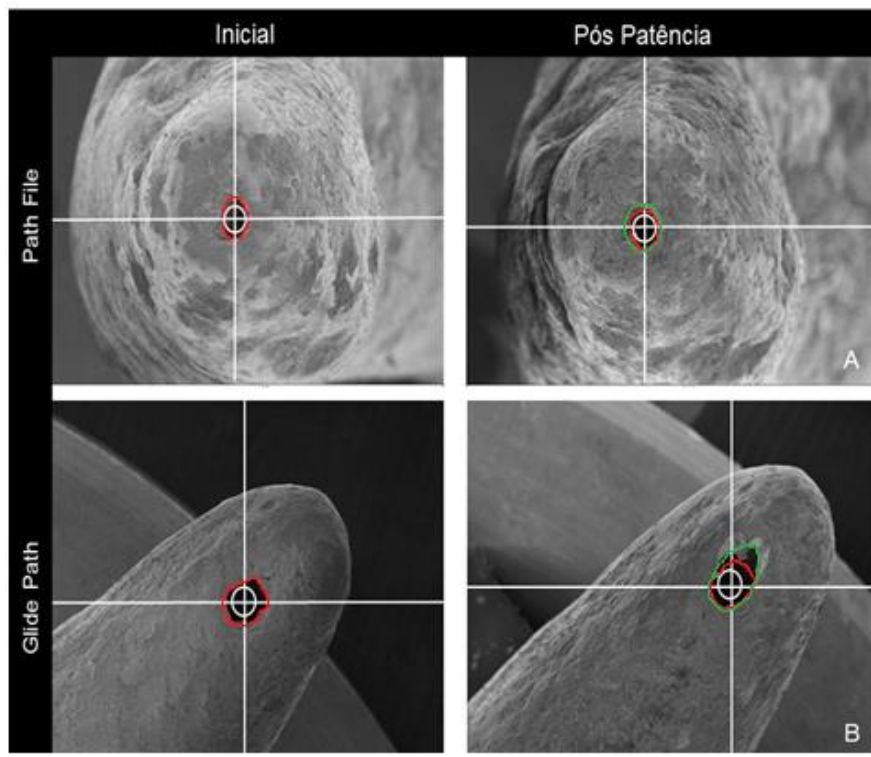

Figura 1: Imagem representativa dos diferentes grupos, onde podemos observar a delimitação inicial do forame apical (vermelho) e após o uso das limas de patência (verde). AGrupo 1 Path file; B- Grupo 2 Glide Path.

\section{DiscuSSÃo}

Patência apical é uma manobra para melhorar o acesso, limpeza e inativação do material no interior do canal compreendido entre o limite ideal de trabalho determinado pelo clínico e o forame apical, que pode ser de 0,5 a $1 \mathrm{~mm}$, dependendo do critério clínico. A patência é um procedimento realizado durante a instrumentação do canal, na qual é feita a penetração de um instrumento de fino calibre, além do comprimento de trabalho, no canal cementário impedindo o acúmulo de detritos, deixando o forame desobstruído, sem alargá$10^{12,19}$. 
A realização da patência é controversa por suas possíveis consequências biológicas, tais como alargamento acidental do forame principal e danos para os tecidos periapicais. Arias et al. (2009) correlacionaram dor pós-tratamento endodôntico à realização ou não de patência. Demonstraram menores episódios de dor pós-tratamento quando a patência foi realizada em dentes não vitais. Nos casos de sintomatologia dolorosa, a duração foi maior quando a patência foi mantida em dentes com dor prévia ao tratamento. De acordo com estes autores a patência não aumenta a incidência, o grau ou a duração da dor pósoperatória $^{10,20}$

Quando a patência é realizada durante o tratamento de canal em dentes com polpa necrosada, cerca de 93,1\% dos casos não referem nenhum tipo de sintomatologia, assim como em dentes vitais com e sem ampliação foraminal. Ou seja, a patência é um procedimento seguro que pode ser aplicada tanto em casos de polpa viva ou necrosada, resultando em uma melhor desinfecção do sistema de canais radiculares e aumentando os índices de sucesso do tratamento endodôntico ${ }^{21}$.

Admite-se, atualmente, que a patência apical ajuda na ação de irrigantes, antissépticos e coadjuvantes para alcançar a zona foraminal, e facilita a remoção de detritos produzidos pela instrumentação, reduzindo consequências mecânicas e biológicas desagradáveis que influenciam o prognóstico do tratamento. $\mathrm{O}$ estudo de Tsesis et al. (2008) não demonstrou diferenças significativas após o uso de uma lima de patência no grau de transporte apical, mostrando uma tendência de manutenção do comprimento de trabalho quando foi realizado a patência apical ${ }^{19,21}$.

Além da patência, a ampliação foraminal torna-se uma alternativa no preparo endodôntico, uma vez que a insuficiente limpeza da região apical pode permitir que placas de micro-organismos continuem presentes nessa região, acarretando insucesso do tratamento endodôntico. De acordo com alguns estudos microbiológicos, quanto maior a preparação apical, maior a redução das bactérias remanescentes. No entanto, Akhlaghi et al. ${ }^{22}$ em estudo realizado in vitro entre dentes tratados, observaram que não houve diferenças significativas em relação à redução de bactérias intracanal em diferentes tamanhos de diâmetros apicais ${ }^{23,24}$.

No presente estudo, os canais foram preparados realizando a patência apical, pois ao adotar essa conduta durante o tratamento clínico do sistema de canais radiculares, reduz-se significativamente a contagem de bactérias do terço apical e previne-se o acúmulo de debris pulpares e dentinários que podem causar bloqueios, desvios, transportes e perfurações no canal radicular $^{12,25}$.

A hipótese nula do presente trabalho foi rejeitada, uma vez que os dois sistemas apresentaram diferença estatisticamente significante quando comparado ao alargamento foraminal após a instrumentação. Ao se comparar os valores das áreas antes e após a instrumentação nos grupos 1 e 2, observou-se que houve diferença estatística $(\mathrm{p}<0,05)$ evidenciando que as limas rotatórias utilizadas neste estudo atingiram o objetivo de ampliar o forame, porém sem diferença entre os grupos analisados.

Neste estudo, o forame apical apresentou desvios, em ambos os sistemas testados. No entanto houve um maior desvio apical no grupo instrumentado com o sistema rotatório Easy \#25.01 (Easy®) em relação ao sistema PathFile (Dentsply Maillefer $\left.{ }^{\circledR}\right)$. Isto pode estar relacionado ao fato de que o instrumento exerce maior pressão na porção externa da parede do canal nas regiões mais curvas coincidentes com o terço apical. O desvio apical é uma iatrogenia de ocorrência comum em canais radiculares com curvatura acentuada, por consequência principalmente da complexidade anatômica da raiz, da flexibilidade do instrumento e do emprego de técnicas de instrumentação inadequadas ${ }^{26,27}$.

As limas de NiTi possuem uma maior flexibilidade do que as limas de aço inoxidável e diversos estudos prévios demonstraram que as limas de NiTi estão associadas a um menor desvio apical quando comparadas com as limas de aço inoxidável. Quanto à deformação do forame, que ocorre em manobras cirúrgicas de desobstrução, não foram encontradas diferenças significativas entre as limas manuais de aço inoxidável ou limas rotatórias de níquel titânio ${ }^{28}$.

Optou-se em realizar a instrumentação rotatória no limite do forame apical, pois durante esse preparo, 
estabelece-se uma melhor trajetória do conduto, pois sabemos a dificuldade de controle do instrumento em seu comprimento real de trabalho, interferindo na limpeza do conduto radicular e consequentemente no processo de reparo. Apesar dos melhores resultados obtidos pelo sistema PathFile, os dois grupos apresentaram desvio em relação a lima inicial, demonstrando que os instrumentos endodônticos acionados a motor ainda necessitam de evolução e mais estudos para o melhor desempenho possível na terapia endodôntica.

\section{Conclusão}

Nas condições do presente estudo, foi possível observar um menor desvio apical para o grupo no qual foi realizado a instrumentação utilizando o sistema Pathfile em relação ao grupo que foi instrumentado pelo sistema EasyLogic. No entanto não houve diferença quanto ao alargamento foraminal entre os grupos analisados.

\section{REFERÊNCIAS}

1. Baugh D, Wallace J. The role of apical instrumentation in root canal treatment: a review of the literature. J Endod. 2005; 31(5):333-40.

2. Sjögren U, Hagglund B, Sundqvist G, Wing K. Factors affecting the long-term results of endodontic treatment. $J$ Endod. 1990; 16(10):498-504.

3. Simon JH. The apex: how critical is it? Gen Dent. 1994; 42(4):330-4.

4. Shuping G, Orstavik D, Sigurdsson A, Trope M. Reduction of intracanal bacteria using nickel-titanium rotary instrumentation and various medications. J Endod. 2000; 26(12):751-5.

5. Ricucci D, Siqueira JF Jr. Biofilms and apical periodontitis: study of prevalence and association with clinical and histopathological findings. J Endod 2010; 36(8):1277-88.
6. Monsef M, Hamedzadeh K, Soluti A. Effect of apical patency on the apical seal of obturated canals. J Endod. 1998; 24(4):284.

7. Camões IC, Salles MR, Fernando MV, Freitas LF, Gomes CC. Relationship between the size of patency file and apical extrusion of sodium hypochlorite. Indian J Dent Res. 2009; 20(4):426-30.

8. Buchanan LS. Management of the curved root canal. $J$ Calif Dent Assoc. 1989; 17(4):18-25.

9. Goldberg F, Massone EJ. Patency file and apical transportation: an in vitro study. J Endod. 2002; 28(7): 510-1.

10. Arias A, Azabal M, Hidalgo JJ, de la Macorra JC. Relationship between post endodontic pain, tooth diagnostic factors, and apical patency. J Endod. 2009; 35(2):189-92.

11. Souza, RA. The importance of apical patency and cleaning of the apical foramen on root canal preparation. Braz Dent J. 2006; 17(1):6-9.

12. Tsesis, I. Amdor B, Tamse A, Kfir A. The effect of maintaining apical patency on canal transportation. Int Endod J. 2008; 41(5):431-5.

13. Schäfer E, Florek H. Efficiency of rotary nickel-titanium K3 instruments compared with stainless steel hand KFlexofile. Part 1. Shaping ability in simulated curved canals. Int Endod J. 2003; 36(3):199-207.

14. Meireles DA, Marques AAF, Garcia LFR, Garrido ADB, Sponchiado EC. Assessment of apical deviation of root canals after debridement with the Hybrid, ProTaper and Path File systems. J Interdiscip Dentistry.2012;2(1):20-4.

15. de Menezes SEAC, Batista SM, Lira JOP, de Melo Monteiro GQ. Cyclic Fatigue Resistance of WaveOne Gold, ProDesign R and ProDesign Logic Files in Curved Canals In Vitro. Iran Endod J. 2017;12(4):468-473.

16. De-Deus G, Belladonna FG, Souza EM, Alves V de O, Silva EJ, Rodrigues E, et AL. Scouting Ability of 4 Pathfinding Instruments in Moderately Curved Molar Canals. J Endod. 2016;42(10):1540-4.

17. Rocha DGP, Alves VO, Martin AS, Fontana CE, Cunha RS, Bueno CES. Comparação entre os sistemas rotatórios Pathfile + Protaper Universal e Twisted Files quanto à preservação da morfologia e ao tempo de trabalho despendido na preparação de canais curvos. Rev Odontol UNESP. 2013; 42(2): 99-103.

18. Silva JM, Brandão GA, Silva EJ, Zaia AA. Influence of working length and foraminal enlargement on foramen morphology and sealing ability. Indian J Dent Res. 2016; 27(1):66-72. 
19. Lambrianidis T, Tosounidou E, Tzoanopoulou M. The effect of maintaining apical patency on periapical extrusion. J Endod. 2001; 27(11):696-8.

20. Ricucci D. Apical limit of the root canal instrumentation and obturation, part I Literature review. Int Endod J. 1998; 31: 384-93.

21. Silva EJ, Menaged K, Ajuz N, Monteiro MR, CoutinhoFilho TS. Postoperative pain after foraminal enlargement in anterior teeth with necrosis and apical periodontitis: A prospective and randomized clinical trial. J Endod. 2013; 39(2):173-6.

22. Akhlaghi NM, Rahimifard N, Moshari A, Vatanpour M, Darmiani S. The effect of size and taper of apical preparation in reducing intra-canal bacteria: a quantitative SEM Study. Iran Endod J. 2014; 9(1):61-5.

23. Nair PN. On the causes of persistent apical periodontitis: a review. Int Endod J. 2006; 39(4):249-81.

24. Paranjpe A, de Gregorio C, Gonzalez AM, Gomez A, Silva Herzog D, Pina AA, et al. Efficacy of the selfadjusting file system on cleaning and shaping oval canals: a microbiological and microscopic evaluation. $J$ Endod. 2012; 38(2):226-33.

25. Flanders DH. Endodontic patency. How to get it. How to keep it. Why it is so important. N Y State Dent J. 2002; 68(6):30-2.

26. Loizides A, Eliopoulos D, Kontakiotis E. Root canal transportation with a $\mathrm{Ni}-\mathrm{Ti}$ rotary file system and stainless-steel hand files in simulated root canals. Quintessence Int. 2006; 5(37):369-74.

27. Aydin C, Inan U, Yasar S, Bulucu B, Tunca YM. Comparison of shaping ability of race and hero shaper instruments in simulated curved canals. Oral Surg Oral Med Oral Pathol Oral Radiol Endod. 2008; 105(3):92-7.

28. Gergi R, Rjeily JA, Sader J, Naaman A. Comparison of canal transportation and centering ability of twisted files, Pathfile-ProTaper system, and stainless-steel hand Kfiles by using computed tomography. J Endod. 2010; 36(5):904-7. 